\title{
Isolation and characterization of Panax ginseng geranylgeranyl-diphosphate synthase genes responding to drought stress
}

\author{
Shadi Rahimi • Yu-Jin Kim • Balusamy Sri Renuka Devi • \\ Altanzul Khorolragchaa • Johan Sukweenadhi • Deok-Chun Yang
}

(C) Koninklijke Nederlandse Planteziektenkundige Vereniging 2015

\begin{abstract}
Geranylgeranyl-diphosphate synthases (GGDPS) catalyze branch point enzymatic reactions producing isoprenoid-derived products which are necessary for plant growth and responses to a wide range of biotic and abiotic stresses. In our study, full length geranylgeranyl-diphosphate synthase 1 (PgGGDPS1) and 2 (PgGGDPS2) cDNA were isolated and characterized from the flower of Panax ginseng and 4-year old $P$. ginseng cv. Gumpoong. The cDNA had open reading frame of 1032 and $1116 \mathrm{bp}$ with a deduced amino acid sequence of 343 and 371 residues for GGDPS1 and GGDPS2, respectively. The calculated molecular mass of GGDPS1 and GGDPS2 were approximately 37.66 and $40.21 \mathrm{kDa}$ with a predicated isoelectric point of 5.32 and 6.23 and predicted localization of plastid. A GenBank Blast X search revealed that the deduced amino acid of $P g G G D P S 1$ shared a high degree of homology with GGDPS from Panax notoginseng. The transcription pattern of GGDPS genes was different at various
\end{abstract}

Y.-J. Kim • D.-C. Yang $(\bowtie)$

Department of Oriental Medicinal Biotechnology, College of

Life Sciences, Kyung Hee University, Yongin 446-701,

Republic of Korea

e-mail: dcyang@khu.ac.kr

S. Rahimi $\cdot$ Y.-J. Kim • B. S. R. Devi • A. Khorolragchaa

J. Sukweenadhi $\cdot$ D.-C. Yang

Graduate School of Biotechnology and Ginseng Bank,

College of Life Sciences, Kyung Hee University,

Yongin 446-701, Republic of Korea developmental stages. Both GGDPS genes were highly expressed in aerial parts of the plant, especially in rapidly growing tissues such as 4-year old flower and stem tissues. Transcript level of $P g G G D P S 1$ was differentially induced in ginseng not only during Pseudomonas syringae pv tomato infection but also after exposure to abiotic stresses. Our results suggested that the induction of GGDPS genes specifically PgGGDPS1 by drought stress may affect chlorophyll levels, intracellular GA content and accumulation of carotenoids as the precursor for higher production of $\mathrm{ABA}$ and possibly stomatal closure as the barrier for water loss.

Keywords Drought - Gene characterization . Geranylgeranyl diphosphate synthase $\cdot$ Organ Expression · Panax ginseng

\section{Abbreviations}

ABA abscisic acid

CLD chain length determination

EST expressed sequence tags

GA3 gibberellin

GGDP geranylgeranyl diphosphate

GGDPS geranylgeranyl-diphosphate synthase

GGR geranylgeranyl reductase

MJ methyl jasmonates

RT- reverse transcriptase-polymerase chain

PCR reaction

SA salicylic acid

YE yeast extract 